\title{
L’ambiguità del teonimo poliano Natigay-Načigay
}

\author{
Elisabetta Ragagnin \\ (Freie Universität Berlin, Deutschland/Università Ca' Foscari Venezia, Italia)
}

\begin{abstract}
This contribution offers an in-depth discussion of the Polian theonym Natigay-Načigay, highlighting its relation with theonyms and shamanistic terms occurring in the European travel literature and/or in Turkic and Mongolic sources.
\end{abstract}

Keywords Mongolian philology. Altaic philology. Etymology. Marco Polo Studies. Shamanism.

Il Devisement dou monde offre preziose informazioni riguardo al mondo spirituale dei Ta(r)tari, ${ }^{1}$ a cui Marco Polo dedica un ricco e ampio capitolo» a un Dio alto, sublime e celeste - il cui nome, noto in altre fonti, era tenggeri $-^{2}$ il viaggiatore veneziano descrive un'altra divinità:

La legge et fede de' Tartari è tale: dicono esservi il Dio alto, sublime et celeste, al qual ogni giorno col torribolo et incenso non dimandano altro se non buon intelletto et sanità; ne hanno poi un altro che chiamano Natigay, ch'è a modo di una statua coperta di feltre o vero di altro, et ciascheduno ne tiene uno in casa sua. Fanno a questo dio la moglie et figliuoli, et pongongli la moglie dalla parte sinistra et li figliuoli avanti di lui, quali pare che li facciano riverenza. Questo dio lo chiamano dio delle cose terrene, il quale custodisce et guarda i loro figliuoli et conserva le bestie et le biade, al quale fanno grande riverenza et honore; et sempre quando mangiano toglieno della parte delle carni grasse, et con quelle ungono la bocca del dio, della moglie et de' figliuoli; dapoi gettano del brodo delle carni fuor della porta agli altri spiriti. Fatto questo, dicono che 'l loro dio con la sua famiglia ha havuto la parte sua, et poscia mangiano et bevono a lor piacere. (R I 46 1-4)

1 Sul termine 'ta(r)taro', impiegato nel Medioevo per designare i Mongoli, cf. de Rachewiltz 1971, Golden 1992, Tatár 2003, Atwood 2004, 528-30.

2 L'etimologia di tenggeri, corrispondente alla forma antico turcica teAltro carattere misteriosoyri - documentata nelle iscrizioni dell'Orkhon (VIII sec.) - è tuttora dibattuta. Per una panoramica etimologica e per una possibile origine paleo-siberiana del termine cf. Georg 2001.

3 Do di seguito il testo di F LXIX 2-6, tradizionalmente assunto come pietra di paragone della tradizione poliana: «Et sachiés que la lor loi est tiel: car il ont un lor diu que l'apelent 
Et come habbiamo detto di sopra, questi popoli sono idolatri, et per suoi dei tutti hanno una tavola posta alta nel pariete della sua camera, sopra la qual è scritto un nome che rappresenta Dio alto, celeste et sublime: et quivi ogni giorno con il thuribulo dell'incenso lo adorano in questo modo, che, levate le mani in alto, sbattono tre volte i denti, pregandolo che li dia buon intelletto et sanità, et altro non li dimandano. Dapoi, giuso in terra, hanno una statua che si chiama Natigai, qual è dio delle cose terrene che nascono sopra tutta la terra, et li fanno una moglie et figliuoli, et l'adorano nell'istesso modo, con il thuribulo et sbattendo i denti et alzando le mani, et a questo li dimandano temperie dell'aere et frutti della terra, figliuoli et simil cose. (R II 26 1-2)

Tra le varianti del teonimo Natigay/Natigai, attestato in Ramusio, si registrano le forme Nacygai (F); Nacigay (Fr); Versigay (K); Nacigay (L); Nacigai (P); Natigai (TB); Nagani (V); Nacigai (VA); Natagai (VB), e Naçagay $(\mathrm{Z})$.

Del Dio si parla anche nel capitolo dedicato alla «regione delle Tenebre»; ${ }^{5}$ i paragrafi sulla religione sono soppressi in R III 44, ma possiamo ricorrere al testo di F CCXVI 3:

Or sachiés que il font un lor dieu de feutre et l'apellent Nacigai. Et encore li font moiliere. Et cesti .II. dieu, ce est Nacigai e sa moiere, dient qe il sunt les dieu de tere e qu'il lor gardent les bestes e les bles e toutes lor bien terenes. Il les orent; e quant il vienent qe il menuient aucune bone viandes, il en ongent la boche a lor deu; et funt bien vies come de bestes.

Nacygai, et dient que celle est dieu tereine qe garde lor filz et lor bestes et lor blee. Il li font grant reverence et grant honor, car cascun en tenent en lor maison, car il font cest deu de $\mathrm{f}\{\mathrm{r}\}$ eutre et de dras et le tenent en lor maison; et encore font la moillier de cest dieu et seç filz: la moiere metent de la senestre partie et les filz devant, et le honorent asseç. Et quant vienent a mangier, il prenent de la char grasse et n'oi<n>gent la bouche a cel dieu et a sa feme et a ssez filz. Et puis prenent dou brod e l'espannent dehors la port<e〉 de sa maison. Et quant il o<n>t ce fait, il dient que lor dieu et sa masnee ont eu lor part. Aprés ce, menjuent il et boivent, car sachiés qu'il boivent la<i>t de jumente, mes si voç di qu'il l'adobent en tel mainere ke le senble vin blance et est bone a boire, et l'apellent chemins». Per un rapido schizzo della tradizione manoscritta del Devisement dou monde cf., in questo volume, gli interventi di Samuela Simion e Alvise Andreose.

4 Il capitolo è attestato soltanto in R e, parzialmente, in Z 45 10-13, che presenta però un testo abbreviato: «Habet quilibet in domo sua statuam appensam in pariete camere, que deum altum celestem representat, vel solummodo ibi nomen dei scriptum. Et sic ipsum adorant: et levant quidem manus et dentes simul ter concutientes, ipsum rogant ut eis erroget vitam longam, alacrem et iocundam. Habent etiam in terra deorsum aliam statuam que Naçagay vocatur, deus terenorum, qui se solum habet intromitere de terenis et rerum nasentium super teram. Apud istum deum eius uxor est et filii, etcetera».

5 La provence de Oscurité di F CCXVII. 
Secondo Pelliot (1959-73, 2: 791-2 nota 295) è possibile individuare una relazione etimologica fra il teonimo poliano - in particolare con la forma «natigay», da lui giudicata come la forma autentica e più vicina all'originale -, il toponimo turcico Ötüken, ${ }^{6}$ mong. Edügen 'Terra' - che seguito dall'epiteto eke 'madre' significa 'Madre Terra' -, e Itoga/Utoga 'il Dio dei Tatari' menzionato nella Historia Mongalorum da Giovanni da Pian del Carpine. Quest'ipotesi è stata successivamente ripresa da altri studiosi, tra gli altri da Tucci, Heissig $(1970,411)$ e de Rachewiltz $(2006,430-31)$, diventando l'interpretazione corrente.

In realtà essa presenta alcune difficoltà. Innanzitutto non c'è una perfetta corrispondenza paleografica tra il lemma poliano, le forme turciche e il teonimo attestato da Pian del Carpine. In particolare risulta problematica l'occorrenza, confermata dalla maggior parte della tradizione manoscritta poliana, della consonante alveolare nasale $n$-. Già Pelliot (1959-73, 2: 791) cercò di spiegare la presenza di questa consonante iniziale interpretandola come un errore di trascrizione, ma indicando allo stesso tempo il carattere non del tutto soddisfacente di questa proposta:

it was once misread with an $n$ - belonging to the preceding word [...] and that wrong form was already substituted everywhere in the archetype of all our Mss, but such a solution is too problematic to allow us to change the spelling.

L'occorrenza di tale consonante potrebbe far pensare ad una forma 'nasalizzata' del mong. etügen: molte varietà linguistiche mongoliche e turciche dell'area siberiana, come alcuni dialetti buriati ${ }^{7}$ occidentali e le lingue sayaniche della taiga (cf. Ragagnin 2011), sono caratterizzate dalla presenza di suoni nasalizzati nei rispettivi sistemi fonetici. Si potrebbe quindi ricostruire una protoforma *nitügei che rispecchierebbe la forma mongolica itügen etügen, e che, forse, era in uso presso popolazioni che vivevano

6 Il toponimo Ötüken è documentato in vari passi delle sopraccitate iscrizioni dell'Orkhon senza precise indicazioni geografiche. Nell'opera enciclopedica del XI secolo Dīvān Luyāt at-Turk (= Compendium dei dialetti turchi) compilata dal karakhanide Maḥmūd al-Kāšyarī, il termine Ötüken identifica un toponimo ubicato fra i deserti dei Tatari nelle vicinanze degli Uiguri (Dankoff, Kelly 1982, 159), localizzabile, forse, a nord della catena dei monti Khangai, ossia nella zona compresa fra i fiumi Orkhon, Selenga e Tes; cf. de Rachewiltz $(2004,1004)$, anche per una bibliografia di riferimento. Si tratta della stessa regione in cui sorgeva la capitale mongola, Karakorum.

7 Il buriate è una lingua mongolica attualmente parlata nella omonima repubblica nella Russia orientale, e nei cui territori si trova il lago Baikal. Piccole isole linguistiche buriate sono inoltre presenti nella Mongolia interna in Cina. Per un profilo della lingua buriate, cf. Skribnik 2003. 
nella detta «Regione delle Tenebre». ${ }^{8}$ Non bisogna inoltre dimenticare che nelle lingue turciche e mongoliche è comune la distorsione fonetica dei termini legati alla religione o ad altri 'tabu' (funzione apotropaica).

L'analisi di Mostaert (1957) di un frammento buddhista del 1312, quindi cronologicamente vicino al racconto poliano - in cui è documentata la forma Načigai eke, letteralmente 'Načigai madre' (mong. eke 'madre' v. sopra) - e funzionalmente corrispondente alla Etügen Eke 'Tellus Mater' della Storia Segreta dei Mongoli, ha portato molti orientalisti, filologi e linguisti (fra cui Cardona 1975, 678) a vedere proprio in questo termine l'origine della forma paleografica poliana Načigay, documentata da F e dal codice zeladiano Z. ${ }^{9}$

Secondo questa proposta le varianti poliane con il nesso - $t i$ - potrebbero facilmente essere ricondotte a corruzioni paleografiche, per scambio $c / t$ (cf. Cardona 1975, 678 e Barbieri 2004, 225). L'analisi filologica condotta da Mostaert (1957) sul frammento buddhista permette inoltre di escludere la corrispondenza fra il teonimo poliano e il toponimo turcico Ötüken.

Per quanto riguarda invece il teonimo Itoga/Utoga attestato in Giovanni da Pian del Carpine, sono necessarie alcune precisazioni preliminari. Il lemma occorre nel cap. III 10 della Historia Mongalorum, dedicato alla religione, al concetto di peccato, alle pratiche di divinazione e purificazione e ai riti funebri dei Mongoli:

Divinationibus, auguriis, aruspiciis, veneficiis, incantationibus multum intendunt, et cum a demonibus eis respondetur, credunt quod Deus ipsis loquatur; quem Deum nominant Utoga, sed Comani Kam ipsum appellant, quem mirabiliter timent et reverentur ac ei oblationes offerunt multas et primitias ciborum et potus, et secundum responsum ipsius faciunt universa. (Daffinà et al. 1989, 240-1)

8 Con ogni probabilità, la «Regione delle Tenebre» va identificata nella Yugria, la regione corrispondente alla parte settentrionale del fiume Ob. Nel XIII secolo tali regioni erano verosimilmente abitate da varie popolazioni indigene dedite alla caccia, alla pesca, e forse all'allevamento delle renne, e di ceppo uralico, turcico e mongolico. Una importante fonte di guadagno di queste popolazioni era il commercio di pelli di animali da pelliccia che da lì raggiungevano i mercati delle importanti città sul mar Caspio e sul mar Nero. L'identificazione non è però del tutto sicura: la descrizione geografica e antropologica di Marco Polo si adatta infatti anche alle terre poste più a Oriente.

9 Nell'antica religione dei Turchi e dei Mongoli al Dio uranico tejri (turcico) e tenggeri (mongolico) si affianca la Dea tellurica - turcico yer-sub, letteralmente 'Terracqueo' (da tur. yer 'terra, suolo' e sub 'acqua') e medio mongolico Etügen Eke. Per le frequenti espressioni formulari del binomio Cielo-Terra cf. Barbieri (2004, 226). Accanto al termine yer-sub le fonti antico-turciche documentano la forma umay 'placenta', in uso anche per designare la dea protettrice delle donne e della prole, probabilmente in virtù delle qualità magiche della placenta (Clauson 1972, 164-5); cf. anche Roux de Rochelle 1990, 156-62. Sulla Madre Terra cf. inoltre Harva 1938, 243-9 e Lot-Falck 1956, 168-72. La divinità yer-sub è ancora venerata fra le popolazioni turciche altai nella Siberia meridionale, e sembrerebbe che questo culto sia di origine iranica (Harva 1938, 245). 
Come già indicato da Daffinà $(1989,416)$, l'identità fra Itoga/Utoga e il $\mathrm{kam}$ dei Comani dipende da un'errata informazione del frate francescano: in cumano infatti il termine qam è usato in riferimento allo sciamano, mentre il lemma che designa Dio è tejri/tegeri (Grønbech 1942, 241, 191). Il termine qam è attestato in turcico antico: qam 'sorcerer, soothsayer, magician'10 (Clauson 1972, 625) e anche in ciagataico: kam (قام) (mongol) 'sorcier; médicin, expérimente' (Zenker 1866-76, 684). Esistono inoltre vestigie in alcune lingue turche moderne, eg. tuvino e khakass xam, con lo stesso significato.

Sul piano etimologico Itoga/Utoga ${ }^{11}$ sembrerebbe originare da turcico ïdu $q$ - aggettivo deverbale da ï - 'mandare, spedire' il cui significato letterale è 'mandato/spedito', ma che già in turcico antico significa 'dedicato a Dio' e 'sacro' (Clauson 1972, 46). L'aggettivo ïduq significa sia 'sacro' che 'cosa sacra'. Alla possibile domanda del francescano «Che cos'è questo?», la risposta potrebbe essere stata semplicemente ïduq, cioè 'cosa sacra', da cui Itoga 'Dio'.

Un ulteriore rischio di fraintendimento è anche causato dalla facile confusione con il termine mongolo udayan 'donna sciamana' (Daffinà 1989, 416), che va probabilmente ricondotto alla stessa radice etimologica della forma turcica appena ricordata (Janhunen 1986, 101). ${ }^{12}$

Una volta stabilito che la forma «Nacigay» corrisponde senza difficoltà al mongolo «Načigay», mentre le varianti del tipo «Natigai», attestate nella tradizione, vanno ricondotte all'attività dei copisti, resta da risolvere la questione legata al sesso della divinità: nel Devisement dou monde infatti Nacigai/Natigai è una divinità maschile, dotata di consorte e prole, ma nella religione mongola Načigai è indubbiamente una figura femminile (cf. anche Barbieri 2004, 226-7).

L'aporia è stata messa in evidenza da molti commentatori; già Yule (Yule, Cordier 1903, 258) tentava di appianare i dati contraddittori spiegando il poliano «Natigai» in base alle forme Nougait o Nogat o Ongotui, documentate nelle relazioni odeporiche del XIX secolo all'interno di alcune delle lingue parlate nelle regioni siberiane limitrofe ai Mongoli quali il

10 Per una descrizione delle funzioni sociali e rituali dello sciamano, intermediario fra il mondo degli spiriti e quello degli uomini, rimandiamo a Harva $(1938,449-561)$ e Eliade (2004, 181-258).

11 Nella Hystoria Tartarorum, come già sottolineato da Daffinà $(1989,416)$, è attestata per 'Dio' la forma paleografica Codar che ha sicuramente origine dalla forma persiana khoda 'Dio'. Nelle lingue turche siberiane Altai e Khakass ci sono tuttora tracce di questo termine: Altai kuday 'Dio' (Verbickij 2005, 150a) e Khakass xuday 'id.' (Baskakov 1953, 290b). Si osservi inoltre che Mandevil'skii - citato in Banzarov $(1891,17)$ e poi in Lot-Falck (1956, 162) - riporta un'ulteriore forma paleografica per Itoga/Utoga: Iroga.

12 Anche il termine mongolico böge 'sciamano' è di origine turcica: $\leftarrow$ tur. bögü 'sage, wizard'; cf. Choi 1992. 
tunguso (il cui territorio attualmente si estende dall'attuale Buriazia alla repubblica yakuta). Tali forme chiaramente si riconducono al mongolo ongyon, oggetto di culto che ricopre particolare importanza nella cornice religioso-spirituale dell'Alta Asia. Con il termine ongyon $^{13}$ si fa riferimento allo spirito protettore della famiglia/clan nella cui abitazione esso si trova. Essi sono concretamente rappresentati attraverso immagini sacre e simulacri, fabbricati solitamente con panno di feltro, ma anche di seta, legno o bronzo. Essi presentano talvolta una forma antropomorfa, motivo per il quale alcuni viaggiatori li hanno paragonati a pupazzi e/o bambole, e 'ospitano' spiriti di vario genere: lo spirito degli antenati, di importanti sciamani deceduti, ma anche cacciatori, pescatori ed eroi che hanno protetto, in vita, la famiglia o il clan dagli attacchi di spiriti ostili. Ongyon possono inoltre essere diversi animali, gli spiriti dell'acqua e della terra nonché i corpi celesti (Harva 1938, 373; Manžigeev 1978, 62-3); ${ }^{14}$ così come divinità o spiriti venerati, pronunciare il cui nome però è tabu. Brevi informazioni sugli ongyon sono anche riportate nell'opera Jāmic at-tawārikh (= La raccolta delle storie) del ministro e poligrafo ilkhanide Rašìd-ud Dīn. ${ }^{15}$

Lo ongyon è quindi l'immagine terrena dello spirito, e come tale è oggetto da venerare.

La presenza e importanza di tali simulacri è documentata estensivamente anche dai viaggiatori francescani. La descrizione più articolata si legge nella Historia Mongalorum (III 2-3) di Giovanni da Pian del Carpine:

13 Cf. la definizione di Lessing (1995, 614a) per il mongolo classico: ongyun (pl. ongyud) 'shamanist spirit inhabiting a material object, genie, guardian spirit, tutelary deity, spirit of a deceased person, ghost; pure, holy, sacred, consacrated; tomb of a saint or eminet person, family tomb; shamanist ancestral idol; naturally white hair' e Kowalewski (1844, 353) ojgun 'pur, saint; Ongon, génie tutélaire d'un endroit; tertre sépulcral, cimetière d'une famille régnante; mets offerts aux manes'; Calmucco ojgon 'heilig, göttlich' (Ramstedt 1935, 287a), buriate ongon 'izobraženie kokogo-l. božestva, dux genij (u šamanistov), idol' (Čeremisov 1973, 356), mongolo Ordos oygon 'saint; endroit de sépulture d'un personnage éminent où se pratique un culte public; sacré, consacré à une divinité, à l'obo' (Mostaert 2009, 514b), daguro ongore 'the shaman's spirit, a departed ancestor' (Martin 1961, 202), e Yakuto oyonńor (? $\leftarrow$ mong.) 'Alter, Greis' (Stachowski 1993, 189).

14 Harva $(1938,376)$ descrivendo gli ongod buriati precisa che nella yurta erano presenti degli speciali ongod che in qualità di spiriti protettivi aiutavano le donne nel parto, e proteggevano la prole e il bestiame. Inoltre i Buriati avevano degli ongod anche all'esterno della yurta. Per gli spiriti protettori del bestiame costruivano infatti piccole casette, dei rifugi, dove essi si potevano riparare in caso di cattivo tempo. Veneravano anche certi monti, dato che secondo loro degli sciamani erano sepolti lì ed esercitavano il loro potere in quella zona circoscritta (foto degli ongyod sono riportate in Harva 1938 alle pp. 373-7).

15 «To each of the twenty four he assigned an animal to be their totem (ongqun)»; e, con riferimento al Clan Suqai'ut, un sottoclan dei Ba'arin: «they say that Genghis Khan turned loose a man off the Ba'arin clan as ongqun, just as they make horses and other animals ongqun, meaning that no one should appropriate it and that it is free and tarkhan [immune]» (si cita dall'edizione Thackstone 1998-9, 1: 105-6). 
Nichilominus habent idola quedam de filtro ad imaginis hominis facta, et illa ponunt ex utraque parte ostii stationis, et subtus illa ponunt quiddam de filtro in modo uberum factum, et illa credunt esse pecorum custodes, ac eis beneficium lactis et pullorum prestare. Alia vero faciunt de pannis sericis et illa multum honorant. Quidam ponunt illa in pulchro curru tecto ante ostium stationis, et quicumque in illo curru aliquid furatur, sine ulla miseratione occiditur. Sed quando volunt illa idola facere, omnes maiores domine conveniunt, que sunt in stationibus illis, et cum reverentia faciunt illa; et cum fecerint interficiunt ovem et illam manducant, et ossa eius igne comburunt. Et cum etiam puer aliquis infirmatur predicto modo faciunt idolum, et ligant super lectum ispius. Duces, millenarii et centenarii hyrcum semper habent in medio stationis.

Predictis vero idolis offerunt primum lac omnis pecoris et iumenti. Et quando primo comedere vel bibere incipiunt, primo eis offerunt de cibariis vel de potu. Et quando aliquam bestiam interficiunt, offerunt cor idolo, quod est in curru, in aliquo cipho, et usque mane dimittunt, et tunc auferunt de presentia eius et decoquunt et manducant. Primo etiam imperatori fecerunt idolum quod ponunt in curru ante stationem honorifice, sicut vidimus ante ordam imperatoris istius, cui offerunt munera multa; equos etiam offerunt ei, quos nullus audet ascendere usque ad mortem. Alia etiam animalia eidem offerunt; que si occidunt ad manducandum, nullum os confringunt ex eis sed igni comburunt. Ei etiam ad meridiem tamquam Deo inclinant, et inclinare faciunt aliquos nobiles qui se reddunt esidem. (Daffinà, Lungarotti, Menestò, Petech 1989, 236-7)

Alcuni dettagli di questa descrizione sono comuni all'Itinerarium di Guglielmo di Rubruck (II 7-8), che si differenzia da Giovanni da Pian del Carpine soprattutto per il tono «irridente»: per designare il feticcio si usano termini sostanzialmente spregiativi come puppa (bambola, pupazzo) e statuuncula (non statua!), e l'idolo più potente, quello al centro della sala, è descritto come una bambola paruula et (?) macilenta (Chiesa 2011, 349):

Et super capud domini est semper una ymago quasi puppa uel statuuncula de filtro, quam uocant fratrem domine, affixe parieti; et superius inter utramque illarum est una paruula, macilenta, que est quasi custos totius domus. Domina domus ponit ad latus suum dextrum ad pedes lecti in eminenti loco pelliculam hedinam impletam lana uel alia materia et iuxta illa, statuuncula, paruulam respicientem uersus famulas et mulieres. Iuxta hostium ad partem mulierum est iterum alia imago cum ubere uaccino, pro mulieribus, que mungunt uaccas: de officio enim feminarum est mungere uaccas. Ad aliud latus hostii uersus uiros est alia statua cum ubere eque, pro uiris, qui mungunt equas.

Et cum conuenerunt ad potandum primo apergunt de potu illi ymagini que est super capud domini, postea aliis ymaginibus per ordinem. Postea 
exit minister domum cum cipho et potu et spargit ter ad meridiem, qualibet uice flectendo genu, et hoc ad reuerentiam ignis; postea ad orientem, et hoc ad reuerentiam aeris; postea ad occidentem ad reuerentiam aque; ad aquilonem proiciunt pro mortuis. (Chiesa 2011, 24-5)

Infine, un breve accenno si trova in Odorico da Pordenone, nel capitolo XXXVI della Relatio, dedicato all'energica azione dei frati minori nella «magna Tartaria»:

Tunc fratres sua idola de feltro accipientes, que ipsi habent, cum cruce et aqua benedicta illa portant ad ignem; deinde omnes de contrata deos suorum vicinorum veniunt videre comburi. Tunc fratres, illa idola accipientes, illa ponunt in ignem et tunc illa de igne exeunt, propter quod fratres postea accipiunt de aqua benedicta, quam in ignem proiciunt, et statim demon fugit ab igne; et sic fratres idolum in ignem proiciunt, ibique comburitur. (Marchisio 2016, 221-2)

Un'ampia documentazione è fornita anche dagli esploratori europei che visitarono queste regioni tra il XVIII e il XX secolo, fra i quali si citano Pallas (1794), Erman (1850), Radloff (1883) e Zelenin (1936), e. Sulle funzioni degli ongyod vedi inoltre Roux de Rochelle (1990, 274-8); Tucci, Heissig (1970, 359-62); Atwood (2004, 423-4); Doerfer (1963, 179-81). Agli ongyod venivano - e vengono tuttora - offerte aspersioni rituali di kumiss, latte di giumenta fermentato; i primi pezzi di cibo durante i pasti; il primo latte delle greggi e delle giumente, ecc., come osservano i viaggiatori medievali nelle testimonianze appena riportate, e come si legge anche nella Storia Segreta dei Mongoli (cap. 193; de Rachewiltz 2006, 37 e 115), dove lo stendardo di guerra viene consacrato con libagioni prima della battaglia. Per tali aspersioni rituali, documentate nella Yuanshi, cronaca della dinastia Yuan, nella sezione relativa alle tradizioni nazionali e agli antichi riti, (cf. Ratchnevsky 1970).

Le indagini sul campo confermano la persistenza di questa pratica: mi diceva Bat - membro della comunità turcofona Dukha ${ }^{16}$ del nord della Mongolia - che lo eeren (forma tuvina per ongyon) è ciò che loro hanno di più prezioso, a cui si rivolgono in presenza di difficoltà di ogni genere. Allo eeren vengono sempre offerte aspersioni rituali. ${ }^{17}$

16 I Dukha sono una piccola etnia turcofona, circa 500 persone, che vivono nelle regioni settentrionale della provincia Khövsgöl in Mongolia. Circa la metà vive ancora allo stato nomade nella taiga e alleva le renne. Per la lingua e storia dei Dukha, cf. Ragagnin 2011.

17 Per una descrizione del cosiddetto sciamanesimo siberiano cf., fra gli altri, Radloff 1893, 1-67; Morgan 1997, 50-3; Harva 1938; Tucci, Heissig 1970; Roux 1990. Fra le popolazioni mongoliche le pratiche sciamaniche si sono meglio conservate fra i Buriati e i Mongoli Darkhat, stanziati nella regione Khövsgöl nella Mongolia settentrionale; cf. Badamxatan 
Il teonimo poliano «Nacigai» sembrerebbe insomma riprodurre la forma medio-mongolica Načigai descritta supra.

Tuttavia la sua maschilità e il fatto che «Nacigai» abbia moglie e figliolanza sembrerebbero frutto di confusione da parte del viaggiatore veneziano, dettata probabilmente da sfumati ricordi di varie forme di ongyod che aveva visto presso i ta(r)tari oltre che, forse, dalle informazioni che aveva ricevute su simili usanze e credenze animistiche di popolazioni limitrofe ai Mongoli dove, forse, veniva venerata una divinità terrena maschile.

A questo proposito vogliamo ricordare che nel pantheon siberiano esistono delle divinità maschili la cui descrizione corrisponde a un "Tellus Pater' (cf. Harva 1938, 243 e 247). Gli Altai della Siberia meridionale, ad esempio, credono in Ülgän o Bai Ülgän, un Dio del bene, antagonista di Erlik, il Dio degli inferi (Verbickij 2005, 412b). Eliade (1964, 198) puntualizza che Ülgän, benché dotato di attributi celestiali, non è del tutto una suprema divinità uranica. Egli è distinto da Tenggri - il Dio-Cielo, supremo Dio uranico delle popolazioni turco-mongole, che nella lingua Altai è Tengere Kairakan $^{18}$ - e possiede piuttosto le caratteristiche di un Dio dell'atmosfera e della fertilità, e ha una consorte e una figliolanza numerosa. La sua funzione è di proteggere la fertilità delle greggi e l'abbondanza dei raccolti.

Si noti infine l'informazione etnografica di Palladius $(1876,15-16)$ citata in Rockhill $(1900,60)$ riguardo agli idoli dei Mongoli daguri e bargu:

the Dahurs and Barhus have in their dwellings, according to the number of the male members, puppets made of straw, on which eyes, eyebrows, and mouths are drawn; these puppets are dressed up to the waist. When someone of the family dies his puppet is taken out of the house, and a new puppet is made for every newly born member of the family. On New Year 's Day offerings are made to the puppets, and care is taken not to disturb them by moving them, etc., in order to avoid bringing sickness into the house.

In conclusione, alla luce di quanto esposto, le informazioni che il Devisement dou monde fornisce su «Nacigai» sembrerebbero riflettere in un'unica divinità vari aspetti del complesso mondo spirituale dell'Alta Asia del XIII secolo, i cui molteplici tasselli non ci sono noti data l'assenza di fonti accurate a riguardo. Tale poliedricità è però documentata, come brevemente esposto in questo saggio, da viaggiatori, etnografi e altri studiosi che a partire dal XIII secolo esplorarono le lontane terre della Russia

1986; Krader 1954, 1975, 1978; Žamcarano 1909; Shimamura 2014; Purev, Purvee 2010; Diószegi 1963.

18 Il termine kairakan è di origine mongolica: cf. mongolo letterario qayiraqan «merciful, gracious; sacred mountain; a formula introducing or concluding a prayer» (Lessing 1995, 914a). 
siberiana e della Mongolia. Benché temporalmente molto più tarde, tali testimonianze, soprattutto quelle che riguardano le popolazioni meno influenzate dal buddismo, sono di grande importanza per meglio capire le informazioni e i fraintendimenti di Marco Polo.

Figura 1. Idolo di stoffa (eeren) Dukha (foto di Elisabetta Ragagnin)

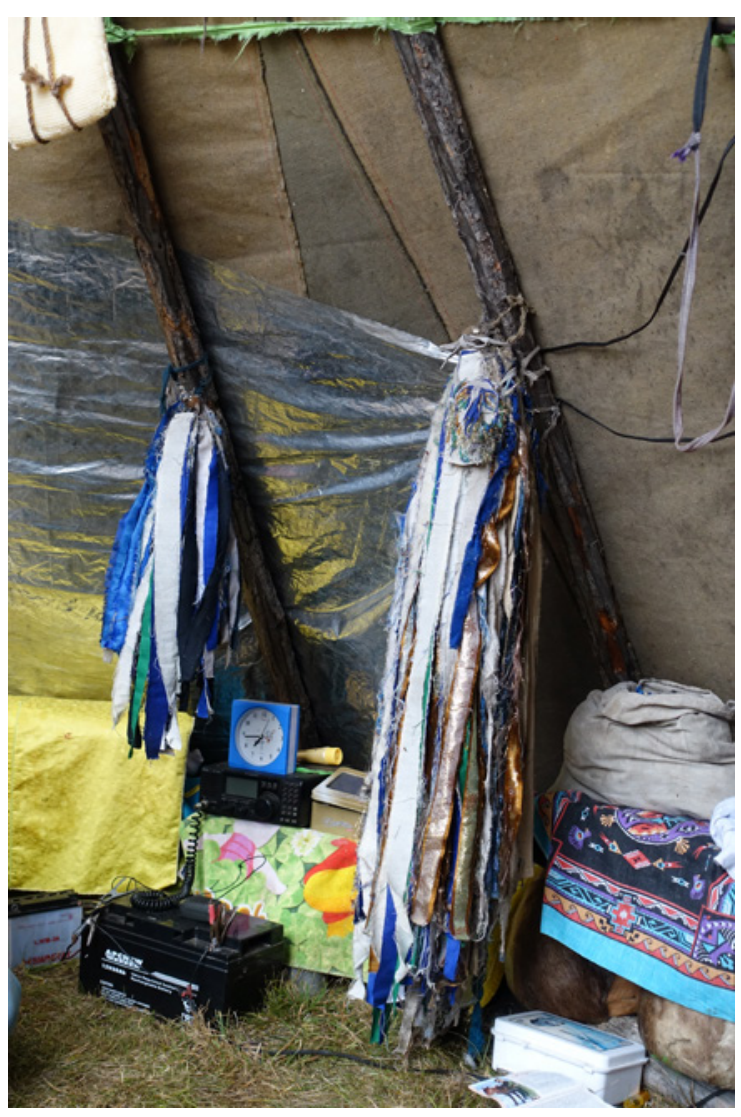

